\title{
Super-carbon spring: a biomimetic design
}

\author{
Dan $\mathrm{Li}^{*}$ and Ling Qiu
}

Low-density cellular materials with high compressibility, elasticity and fatigue resistance hold promise for applications in mechanical damping, flexible electronics, actuators and multifunctional polymer nanocomposites [1-5]. Recently, cellular materials with ultralow density and superelasticity have been successfully synthesized with robust and flexible nanoscale building blocks such as graphene and carbon nanotubes [6-9]. However, when these cellular materials undergo large strain cyclic compression, microstructure cracking or buckling failure often occurs, leading to large energy dissipation, plastic deformation and reduction of strength $[1,2,6]$. As the mechanical properties of a cellular material are dependent on not only the mechanical attributes of the basic building blocks, but also the hierarchical structure of the assembled cellular network, it has been very challenging to find an effective solution to synthesize a cellular material with combined high compressibility, elasticity and fatigue resistance.

Excitingly, a team led by Shu-Hong Yu and Heng-An Wu from the University of Science and Technology of China (USTC) [10] recently reported a new biomimetic design to tackle this long-standing challenge by borrowing a design widely existing in macroscopic biological world. They observed that the arch-shape structure in biology was highly favorable for realization of combined fatigue resistance and elasticity. A good example is the arch of human feet, which acts as an elastic spring-type cushioning system to facilitate human motions (Fig. 1a) and the arch-shaped spring-type suspension systems can help to support the axle and absorb mechanical shock.

The USTC researchers have provided a smart strategy to realize the biomimetic design at the micrometer scale in carbon-based cellular materials. They first fabricated a monolithic chitosan-graphene oxide (CS-GO) composite cellular scaffold consisting of numerous parallel, aligned and thin lamellas through a bidirectional freezing process, followed by freeze drying and thermal annealing. Interestingly, they discovered that the thin lamellas were self-crumpled into waved micro-arch morphology as a result of the local volume decrease of the CS matrix caused by its partial mass loss in the annealing process (Fig. 1b).

The resultant carbon cellular structure containing lamellar micro-arches is found to be highly elastic, despite the fact that the obtained monolithic material is composed of amorphous carbon-graphene (C-G) composite constituent, which is generally very brittle. The carbon elastomer can completely recover to its original shape upon $90 \%$ compression strain with a small energy dissipation of $\sim 0.2$, which is lower than that of all the previously reported values (0.3-0.7). It can rebound a steel ball in spring-like fashion with fast recovery speed of $\sim 580$ $\mathrm{mm} \mathrm{s}^{-1}$ (more than four times of the previously reported maximum value) (Fig. 1c). More interestingly, this carbon elastomer also exhibits a high level of fatigue resistance. It can undergo more than $1.0 \times 10^{6}$ compression cycles at $20 \%$ strain, $2.5 \times 10^{5}$ cycles at $50 \%$ strain and $1.0 \times 10^{4}$ cycles at $80 \%$ strain yet still sustains its structural integrity (Fig. 1d). This is comparable to those of the state-of-the-art macroscopic structural materials composed of strong and robust solid constituents.

In situ SEM observation of the materials under compression process (Fig. 1e) and mechanical simulations (Fig. 1f) reveal that the thin-shell micro-arches do display typical elastic behavior, and can undergo large out-of-plane deformation without yielding because of the small in-plane material strain. Both the experiments and simulations confirm that the excellent elasticity of the monolithic elastomer is indeed originated from the intrinsic elasticity of the micro-arch structural units.

This work presents an excellent example that the design

Monash Centre for Atomically Thin Materials, Department of Materials Science and Engineering, Monash University, Clayton VIC 3800, Australia

*Email: Dan.Li2@monash.edu 
a

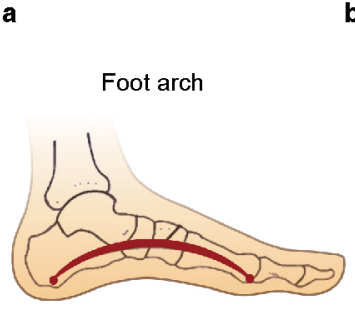

b
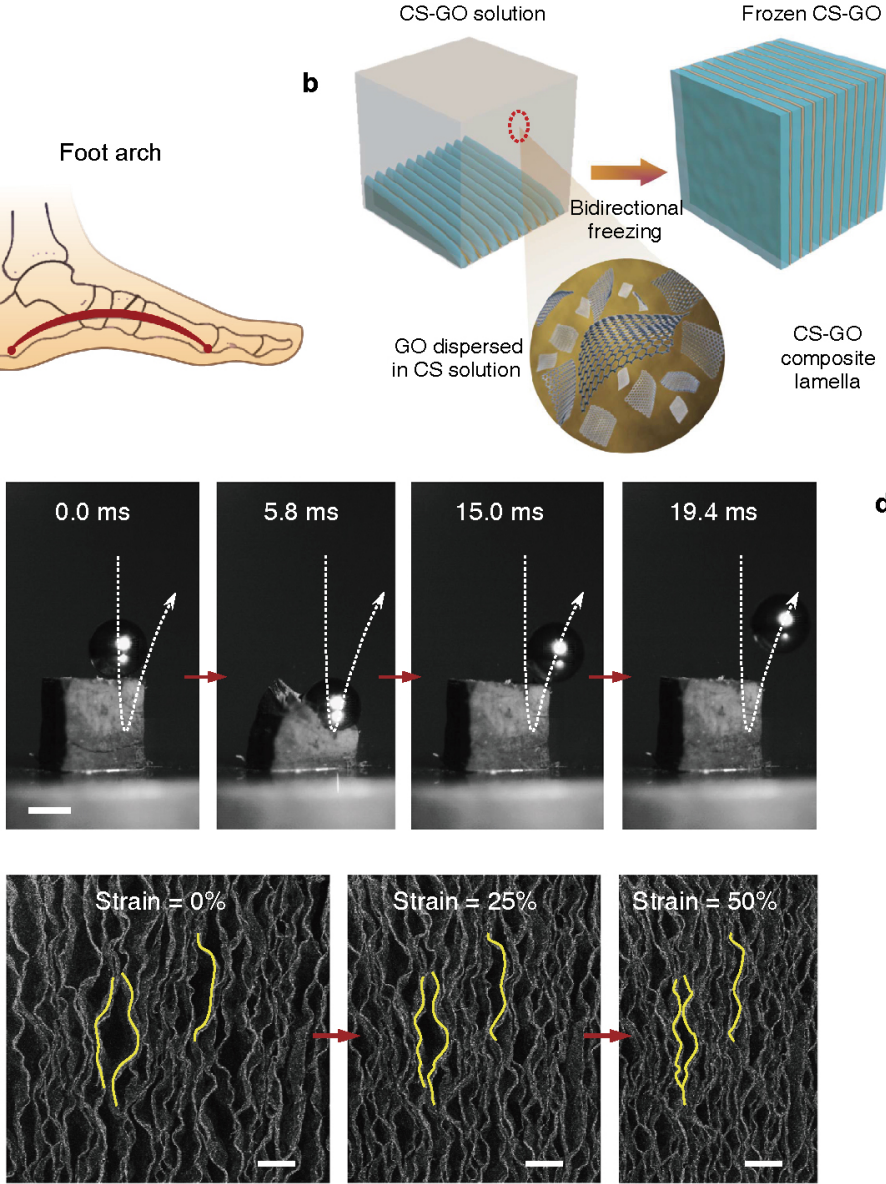
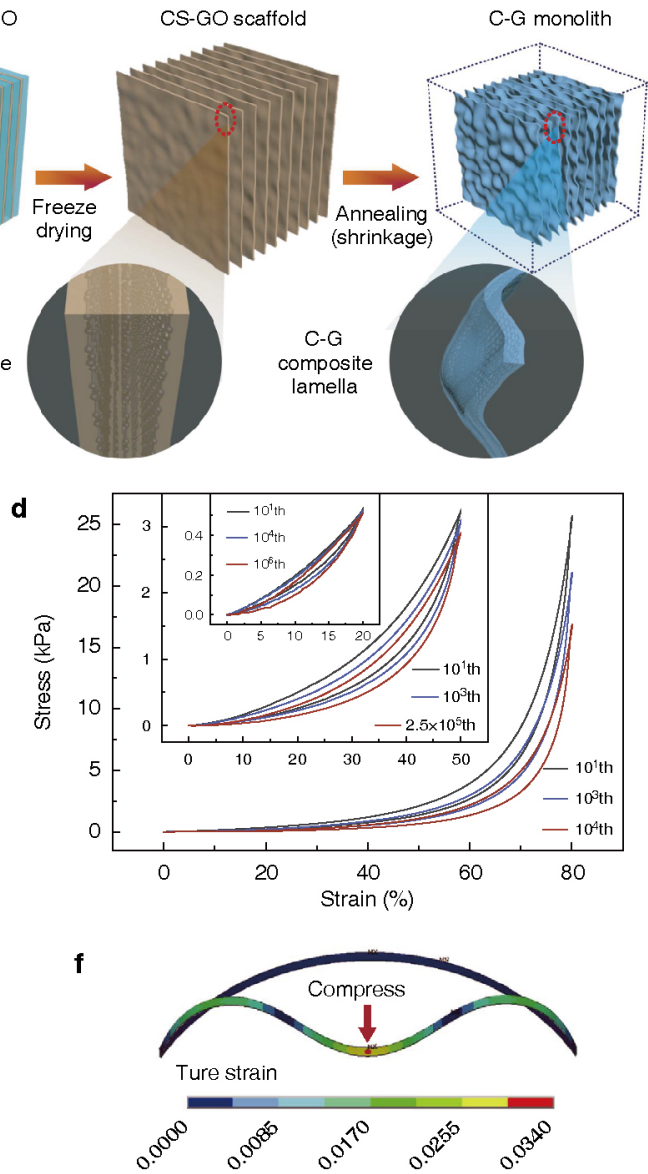

Figure 1 Elastic carbon monolith based on a micro-arch structural design. (a) An example of elastic macro-arch in human feet. (b) Schematic illustration showing the fabrication process of the carbon elastomer. (c) Real-time images from high-speed camera showing that the carbon elastomer can rebound a steel ball at a high speed, in a spring-like fashion. (d) Stress-strain curves of the carbon elastomers at $20 \%$ strain for $1 \times 10^{6} \mathrm{cycles}$, at $50 \%$ strain for $2.5 \times 10^{5}$ cycles and at $80 \%$ strain for $1 \times 10^{4}$ cycles. (e) In situ SEM observation of the carbon elastomer under compression with maximum strain of $50 \%$. (f) The true material strain (von Mises total strain) profiles of the arch-shaped thin-shell model under large geometry deformation. Reprinted with permission from Ref. [10], Copyright 2016, Nature Publishing Group.

principles currently being applied in macroscopic mechanics could be readily transferred to the microscopic cellular materials world. The biomimetic design demonstrated in this work highlights great potential to realize exceptional cellular materials by rational design of both the properties of solid constituents and hierarchical structure. We expect that this work will stimulate more research effort towards creating new generation of functional cellular materials.

Received 29 November 2016; accepted 30 November 2016; published online 28 December 2016

1 Meza LR, Das S, Greer JR. Strong, lightweight, and recoverable three-dimensional ceramic nanolattices. Science, 2014, 345: $1322-1326$

2 Cao A, Dickrell PL, Sawyer WG, et al. Super-compressible foamlike carbon nanotube films. Science, 2005, 310: 1307-1310

3 Wu Y, Yi N, Huang L, et al. Three-dimensionally bonded spongy graphene material with super compressive elasticity and near-zero Poisson's ratio. Nat Commun, 2015, 6: 6141

4 Qiu L, Bulut Coskun M, Tang Y, et al. Ultrafast dynamic piezoresistive response of graphene-based cellular elastomers. Adv Mater, 2016, 28: 194-200

5 Qiu L, Liu D, Wang Y, et al. Mechanically robust, electrically conductive and stimuli-responsive binary network hydrogels enabled by superelastic graphene aerogels. Adv Mater, 2014, 26: 3333-3337

6 Schaedler TA, Jacobsen AJ, Torrents A, et al. Ultralight metallic microlattices. Science, 2011, 334: 962-965

7 Qiu L, Liu JZ, Chang SLY, et al. Biomimetic superelastic graphenebased cellular monoliths. Nat Commun, 2012, 3: 1241

8 Zhu C, Han TYJ, Duoss EB, et al. Highly compressible 3D periodic graphene aerogel microlattices. Nat Commun, 2015, 6: 6962

9 Bi H, Chen IW, Lin T, et al. A new tubular graphene form of a tetrahedrally connected cellular structure. Adv Mater, 2015, 27: 5943-5949

10 Gao HL, Zhu YB, Mao LB, et al. Super-elastic and fatigue resistant carbon material with lamellar multi-arch microstructure. Nat Commun, 2016, 7: 12920 